\title{
Transport rate-based model for overland flow and solute transport: Parameter estimation and process simulation
}

\author{
Zhi-Qiang Deng ${ }^{\mathrm{a}, *}$, João L.M.P. de Lima ${ }^{\mathrm{b}}$, Vijay P. Singh ${ }^{\mathrm{a}}$ \\ ${ }^{a}$ Department of Civil and Environmental Engineering, Louisiana State University, Baton Rouge, LA 70803-6405, USA \\ ${ }^{\mathrm{b}}$ IMAR-Institute of Marine Research, Coimbra Interdisciplinary Centre, Department of Civil Engineering, Faculty of Science \\ and Technology, Polo 2, University of Coimbra, 3030-290 Coimbra, Portugal
}

Received 5 September 2003; revised 7 March 2005; accepted 31 March 2005

\begin{abstract}
A one-dimensional mathematical model, termed a transport rate-based model, is developed for solute transport over infiltrating soil slopes under constant rainfall and declining sources. The model is comprised of (1) the kinematic-wave equation for overland flow, (2) a transport rate-based advection equation for overland solute transport, (3) a moment-based method for estimation of the parameters involved in the flow and solute transport equations, and (4) a semi-Lagrangian algorithm for numerical solution of the solute transport equation. Data from a single soil flume experiment under constant rainfall established the proof-of-concept for this new model. Sodium chloride was applied to the soil surface to simulate the presence of a declining diffuse pollutant. The parameters involved in the flow equation were found to take on the values that correspond to turbulent flow. With these turbulent flow parameters the simulated hydrograph displayed an initial rising limb, followed by a constant flow discharge. The profile of solute concentration exhibits a steep receding limb transitioning into an elongated tail. The solute transport rate follows a non-Gaussian distribution that does not appear to have been derived before. These theoretical hydrographs and pollutographs are in good agreement with those measured in laboratory, demonstrating the laboratory proofof-concept for the transport rate-based model on soil and pavement blocks.
\end{abstract}

(C) 2005 Elsevier B.V. All rights reserved.

Keywords: Overland flow; Solute transport rate; Numerical model; Parameter estimation

\section{Introduction}

Overland flow and concomitant solute transport are a major source of pollutants in receiving surface waters. An important class of these pollutants arise from surface applied or soil-incorporated pesticides,

\footnotetext{
* Corresponding author. Tel.: + 1225578 6850; fax: + 1225578 8652.

E-mail address: zdeng@1su.edu (Z.-Q. Deng).
}

nutrients, and other agricultural chemicals. Solute transfer from soil to surface runoff and subsequent overland solute transport also decrease the efficiency of the applied chemicals.

A sound mathematical model can provide an efficient and economic tool by which a large number of scenarios can be simulated and compared in a short time and then the best alternative of addressing the problems may be found. Consequently, a wide spectrum of models, ranging from simple empirical 


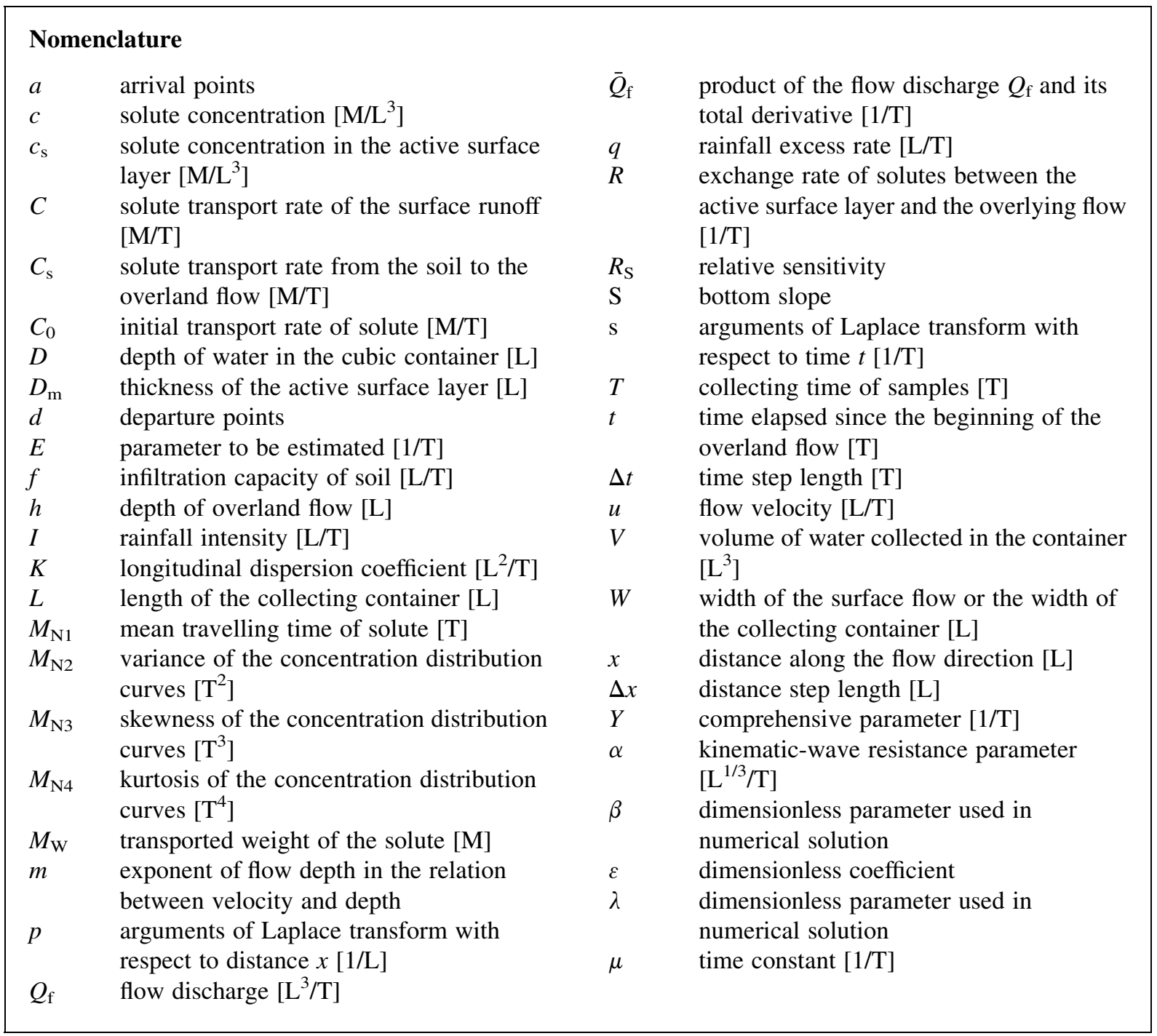

formulas to comprehensive distributed physically and chemically based descriptions (Beven, 1989; Novotny and Olem, 1994; Abbott and Refsgaard, 1996; Govindaraju and Erickson, 1996; Preti, 1999; Wallach et al., 2001; Singh and Woolhiser, 2002; Singh, 2002) have been proposed for prediction of the overland flow and solute transport. These models may also be intended for planning best management practices (BMPs) for efficient utilization of the applied chemicals and for effective protection of the water environment. Some of the models show great promise and have been increasingly used (Singh and
Woolhiser, 2002). Unfortunately, practical applications of the models have long been plagued by three problems associated with (1) parameter estimation, (2) definition of the initial condition, and (3) prescription of the source term.

The first problem of existing simulation models for overland solute transport lies in the lack of a sound method for estimating the parameters involved in the models. Some parameters, such as the exponent and coefficient of flow depth in the momentum equation, are simply taken as empirical constants that can be determined by assuming a laminar flow (Myers, 2002) 
based on the Reynolds number. For more accuracy, these parameters are determined by fitting model simulations to measurements. By calibrating the semiempirical parameters, the simulations either lose predictive capabilities for solving practical problems or at least the simulated values are less certain. Due to the lack of an effective method for parameter estimation in the literature, information about parameters and parameter selection is often discussed in only a cursory and ambiguous manner. In order to fit model simulations to observations, some models introduce purely empirical correction factors that have no physical basis (Akan, 1987). As a result, practicing engineers in particular frequently find that such journal papers are generally not usable for engineering application (ASCE Task Committee on Definition of Criteria for Evaluation of Watershed Models of the Watershed Management Committee, 1993). A sound method for estimation of model parameters is therefore needed so that the parameters can be evaluated in an objective fashion and the models can be put to practical use.

The second problem of existing models is the deviation of the initial concentration distributions from measurements, as shown in Fig. 1. In most field and laboratory measurements, the solute concentration approximately declines exponentially from a first-flush-caused highest initial concentration, instead of the commonly assumed zero value. This is

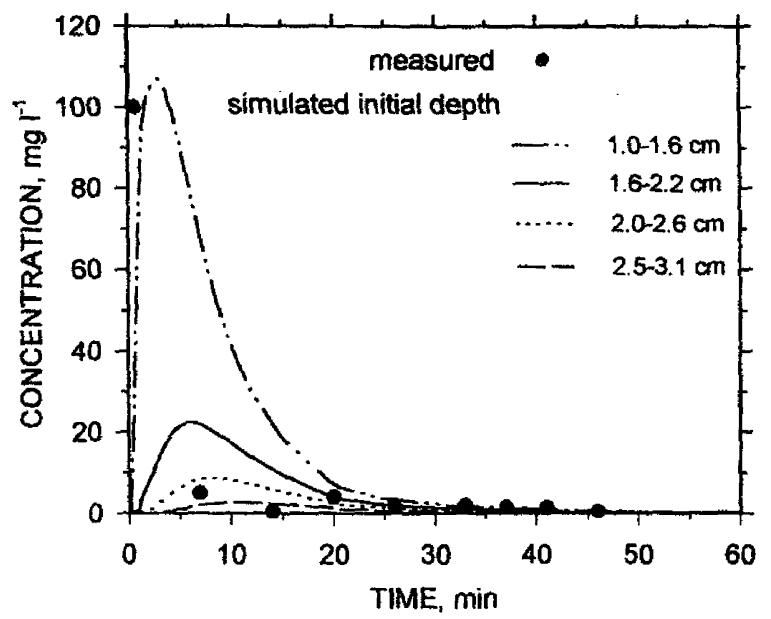

Fig. 1. Measured and predicted nitrate concentration in the overland flow at the catchment outlet [Reproduced from Fig. 5(b) of Wallach et al. (2001) with permission.]. illustrated in Figs. 7, 8, and 12 of Hubbard et al. (1989a) and in Figs. 1 and 6 of Hubbard et al. (1989b). Such a significant disagreement is caused by an inappropriate prescription due to the uncertainty of actual initial concentration conditions. For simplicity and convenience of both the numerical and analytical solutions, a zero initial concentration is often taken for granted. However, common sense clearly dictates that surface pollutants should be continually diluted from a maximum concentration once overland flow occurs from homogeneous land uses on the same soil. Consequently, a zero initial concentration for overland solute transport is impossible. In terms of practical and environmental concerns with overland flow, the highest concentration occurring in the initial runoff is most important and hence should be evaluated as accurately as possible. However, few exist models can make such a simulation. Thus, the solute transport algorithm should be derived from the advection-dispersion equation and have clear and convenient solution conditions for overland solute transport.

The third problem of many existing models is the assumption of an instantaneous diffuse source. This assumption also rarely holds in reality. In watersheds, pollutants are normally trapped in depression zones and stored in the active surface layer and thus gradually transferred to surface runoff during rainfall (Singh, 1997). As indicated in the measurements of Hubbard et al. (1989a and 1989b), an exponential or a similar decline of soil solute can be observed in field and laboratory experiments due to the storage and gradual release of the solute. Improper treatment of the source term may yield significant simulation errors. Actual soil solute loss due to overland flow obviously declines with time during rainfall and such a feature must be embodied in a sound model.

The overall goal of this paper is therefore to develop a new physically based overland flow and solute transport model which is capable of addressing these three problems. The specific objectives are (1) to present an efficient mathematical model for overland flow and solute transport based on the characteristics of solute wash-off on soil surfaces, (2) to develop a moment-based method for estimation of parameters involved in equations of the model using the Laplace transform, (3) to conduct a series of experiments of overland flow and solute transport to collect data 
needed for parameter estimation, (4) to propose an efficient method for solving the equations of the model numerically, and (5) to test the efficacy of the mathematical model and the parameter estimation method using the laboratory data initially. The five specific objectives are presented in the following five consecutive Sections 2-6, respectively.

\section{Overland flow and solute transport equations}

Overland solute transport caused by rainfall is driven by a non-uniform flow with an increasing discharge along the slope. The overland flow and solute transport processes can be mathematically described with two governing equations, although physically the processes are inseparable.

\subsection{Kinematic-wave overland flow equations}

Flow over a pervious plane is generally described by the kinematic wave approximation of the SaintVenant equations, which are a one-dimensional approximation of the laws of conservation of mass and momentum of shallow water flowing longitudinally and infiltrating vertically (Woolhiser, 1975; Singh, 1996). The kinematic wave equation can be expressed on a moderate to steep uniform slope (Martin and McCutcheon, 1999) as

$$
\begin{aligned}
& \frac{\partial h}{\partial t}+\frac{\partial(u h)}{\partial x}=I-f \quad \text { with } \\
& u=\alpha h^{m-1} \text { or } Q_{\mathrm{f}}=\alpha W h^{m}
\end{aligned}
$$

where $h$ is the depth of overland flow, $t$ is time, $u$ is the velocity of the flow, $x$ is the distance along the flow direction, $I$ denotes the rainfall intensity, $f$ stands for the infiltration capacity of soil that is constant for this application, $\alpha$ is the kinematic-wave resistance parameter, $m$ is an empirical exponent, $Q_{\mathrm{f}}$ is the flow rate, and $W$ is the width of the surface flow. Overland flow can be classified as being either laminar or turbulent (de Lima, 1992; Wallach et al., 2001; Myers, 2002), depending on the Reynolds number defined based on the flow depth. The parameters $\alpha$ and $m$ can be derived for both flow regimes such that only one parameter is independent. As a result, parameter $m$ is first assumed to take on the value $5 / 3$ of turbulent flow and parameter $\alpha$ to be an unknown to be determined. If the calibrated value of $\alpha$ is close to that calculated by the Manning equation (SI units) for wide flows, then the existence of turbulent flow is confirmed. Otherwise, the laminar or transitional flow regime may be occurring.

The solution of the kinematic wave equation then requires only initial and upstream boundary conditions (Molen et al., 1995; Singh, 1996). The initial and boundary conditions imposed on Eq. (1a) are specifically

$h(x, 0)=0$ for $0 \leq x<L$

$h(0, t)=0$ for $0 \leq t<\infty$

where $L$ is the length of overland flow along a uniform slope of homogeneous soil. The simulation of overland flow then reduces to the solution of Eq. (1a) subject to Eqs. (1b)-(1d) with one unknown parameter $\alpha$ to be determined by calibration.

\subsection{Overland solute transport equation}

Solute transport is generally described by the advection-dispersion equation (ADE) (Kiely, 1997; Martin and McCutcheon, 1999; Deng, 2002). For overland solute transport, the solute is assumed in this derivation to be uniformly distributed in the uppermost layer of the soil (or the surface active zone or the 'extraction active layer') both horizontally and vertically before the start of rainfall. Based on this concept a diffuse source term is therefore included in the ADE. The change in concentration of the solute in the surface active zone is assumed based on a few observations to be proportional to the difference between the existing and the incoming concentrations in the same soil water volume (Flanagan and Foster, 1989; Preti, 1999). Then, the ADE can be expressed as

$\frac{\partial(c h)}{\partial t}+\frac{\partial(c u h)}{\partial x}=\frac{\partial}{\partial x}\left(K h \frac{\partial c}{\partial x}\right)+R D_{\mathrm{m}}\left(c_{\mathrm{s}}-c\right)$

where $c$ represents the solute concentration or the mass of solute per unit volume of runoff, $K$ denotes the longitudinal dispersion coefficient, and $c_{\mathrm{s}}$ stands for the solute concentration in the active surface 


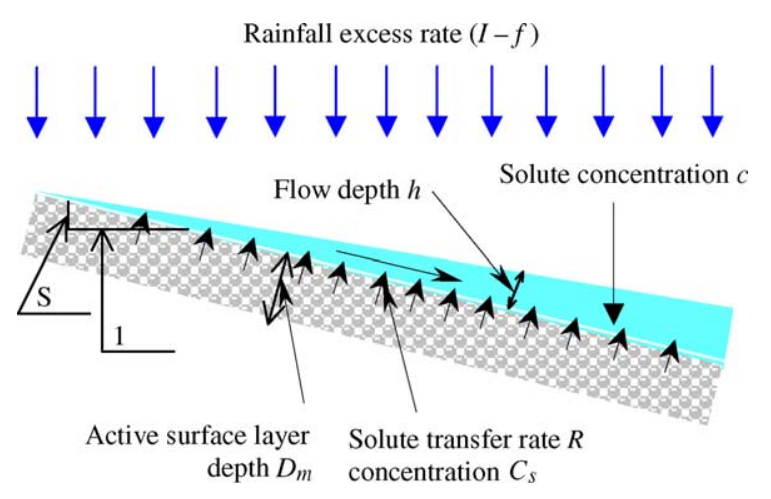

Fig. 2. Conceptual model for overland solute transport.

layer, as shown in Fig. 2. This layer is taken (1) to have a uniform concentration $c_{\mathrm{s}}$, (2) to have a thickness or depth scale of $D_{\mathrm{m}}=\varepsilon h$ that is proportional to the depth $h$ of overland flow with a dimensionless coefficient $\varepsilon$, (3) to act as a declining diffuse source of solute supply to overland flow, and (4) to have a known concentration prior to the beginning of rainfall. The exchange rate of solute $R$ between the active surface layer and the overlying flow has a dimension of $[1 / T]$. The exchange rate can also be termed as transfer rate of solute from the active surface layer to the overland flow because the result of overland solute transport is always the decline of solute content in the soil in the absence of production or creation. This initial selection of $D_{\mathrm{m}}=$ $\varepsilon h$ is mainly used to achieve consistency in dimensions $[L]$. Initially the coefficient $\varepsilon$ can be estimated using calibration with measurements. Later, values of $\varepsilon$ can be statistically correlated with all significant physical parameters for additional insights into a more detailed experimental and theoretical investigation of the representative depth of the active surface layer $D_{\mathrm{m}}$.

For mathematical convenience, Eq. (2a) is recast as

$$
\begin{gathered}
c\left(\frac{\partial h}{\partial t}+\frac{\partial(u h)}{\partial t}\right)+h\left(\frac{\partial c}{\partial t}+u \frac{\partial c}{\partial x}\right) \\
=\frac{\partial}{\partial x}\left(K h \frac{\partial c}{\partial x}\right)+R D_{\mathrm{m}}\left(c_{\mathrm{s}}-c\right)
\end{gathered}
$$

For overland solute transport, the dispersion term is generally negligible as compared to other terms (Abbott and Refsgaard, 1996; Wallach et al., 2001), i.e. $K=0$, then

$\frac{\partial c}{\partial t}+u \frac{\partial c}{\partial x}=R \varepsilon\left(c_{\mathrm{s}}-c\right)-\frac{(I-f)}{h} c$

By incorrectly assigning the initial concentration $c_{0}$ to be either zero (Abbott and Refsgaard, 1996) or to be equal to the initial soil surface concentration (Wallach et al., 2001), Eq. (3a) incorrectly represents the runoff concentration curve characterized temporally by a rising limb followed by a receding limb. To make the solution practical, Eq. (3a) can be changed to a solvable form. To that end, replacing $c$ by $c Q_{\mathrm{f}} / Q_{\mathrm{f}}=C / Q_{\mathrm{f}}$ and $c_{\mathrm{s}}$ by $c_{\mathrm{s}} Q_{\mathrm{f}} / Q_{\mathrm{f}}=\mathrm{C}_{\mathrm{s}} / Q_{\mathrm{f}}$, splitting each differential term of $C / Q_{\mathrm{f}}$ into two terms by taking partial derivatives of the terms, and then multiplying both sides of the equation by $Q_{\mathrm{f}}$, results in

$$
\begin{aligned}
& \frac{\partial C}{\partial t}+u \frac{\partial C}{\partial x}+Q_{\mathrm{f}}\left[\left(\frac{\partial\left(1 / Q_{\mathrm{f}}\right)}{\partial t}+u \frac{\partial\left(1 / Q_{\mathrm{f}}\right)}{\partial x}\right)\right] \\
& C=\varepsilon R\left(C_{\mathrm{s}}-C\right)-\frac{(I-f)}{h} C
\end{aligned}
$$

where $C=c Q_{\mathrm{f}}$ and $C_{\mathrm{s}}=c_{\mathrm{s}} Q_{\mathrm{f}}$ are termed the transport rate of solute by surface runoff and the transport rate of solute from the soil to the overland flow. For simplicity the third term on the left hand side of Eq. (3b) is defined as

$\bar{Q}_{\mathrm{f}}=Q_{\mathrm{f}}\left(\frac{\partial\left(1 / Q_{\mathrm{f}}\right)}{\partial t}+u \frac{\partial\left(1 / Q_{\mathrm{f}}\right)}{\partial x}\right)$

Substituting Eq. (3c) into Eq. (3b) and rearranging the terms yields

$\frac{\partial C}{\partial t}+u \frac{\partial C}{\partial x}=E C_{\mathrm{s}}-Y C$

in which $E=\varepsilon R$ and $Y=(I-f) / h+E+\bar{Q}_{\mathrm{f}}$ are introduced. Eq. (4a) describes the change of solute transport rate $C$ in overland flow due to the net rainfall dilution and solute transfer from the surface active zone and thus is termed, the rate-based solute transport equation. For a steady and net rainfall flow, $\bar{Q}_{\mathrm{f}}$ in Eq. (3c) approaches zero. The commonly used advection equation of solute transport is by derivation a form of Eq. (4a). Consequently, Eq. (4a) is a generalized solute transport equation useful particularly for spatially varied overland flow on moderate to steep slopes. 
Pollutants are generally washed off or transferred to runoff gradually rather than instantaneously (Hubbard et al., 1989a, b). Decline of the solute transfer rate in the active surface layer caused by the transfer of solutes from the soil to the overlying runoff is therefore assumed to follow an exponential law with a time constant $\mu$ of the dimension [1/T], i.e.

$C_{\mathrm{s}}=C_{0} \exp (-\mu t)$

Eq. (4a) is also subject to the following initial and boundary conditions:

$$
C(x, 0)=0 \text { and } C_{\mathrm{s}}(x, 0)=C_{0}(x) \text { for } 0 \leq x<L
$$

$C(0, t)=0 \quad$ for $0 \leq t<\infty$

in which $C_{0}$ is the initial transport rate of solute from the soil to the overland flow. The two parameters $C_{0}$ and $\mu$ are also to be estimated by calibration. Note that $t=0$ refers to the beginning of overland flow instead of the starting of rainfall. Solute transport from the soil to the soil water starts with rainfall and infiltration but there is no overland solute transport until the overland flow begins. Therefore, a zero initial overland transport rate $C_{0}$ with a non-zero initial concentration value of $c(x, 0)$ is reasonable, because $C=c Q_{\mathrm{f}}$ and $Q_{\mathrm{f}}=0$ at $t=0$. As $c=C / Q_{\mathrm{f}}$ and both $C$ and $Q_{\mathrm{f}}$ are zero at $t=0, c(x, 0)$ is thus uncertain. One way to estimate the initial value of $c\left(x, t_{\text {initial }}\right)$ is to take $t_{\text {initial }}=5 \mathrm{~s}$ for solute transport over soils or even a shorter time, for instance, $2 \mathrm{~s}$ for solute transport over urban impervious surfaces, then $c\left(x, t_{\text {initial }}\right)$ can be calculated as $C\left(x, t_{\text {initial }}\right) / Q_{\mathrm{f}}\left(x, t_{\text {initial }}\right)$. The shorter is $t_{\text {initial }}$, the more accurate are the results.

Eqs. (1) and (4) can be employed to simulate overland flow and solute transport as long as the unknown parameters, $\mu, C_{0}, E$ and $\alpha$ are determined. Consequently, proper methods for estimation of the parameters of Eqs. (1) and (4) are essential for the solution.

\section{Parameter estimation}

Parameters may be estimated using various methods, such as the moment method, the maximum likelihood method, the $L$-Moment method, maximum entropy method, and others (Singh, 1998; Doherty and Johnston, 2003). Of all the alternatives, the moment method performs well in most cases and is easy to use (Adrian et al., 2002; Singh and Deng, 2003). The moment method is therefore adopted in this paper for parameter estimation. To that end, the solute transport equation is first transformed by the Laplace method.

\subsection{Laplace transform of the solute transport equation}

In terms of parameter estimation, only Eq. (4) is needed because this equation contains all the unknown parameters to be determined. Using the initial and boundary conditions prescribed as Eqs. (4c) and (4d), the Laplace transform of Eq. (4a) with respect to time $t$ is

$u \frac{\partial \bar{C}(x, s)}{\partial x}+(s+Y) \bar{C}(x, s)=E \bar{C}_{\mathrm{s}}(x, s)$

where $\bar{C}(x, s)$ is the Laplace transform of $C(x, t)$ with respect to $t$. Likewise, Eq. (4b) gives

$\bar{C}_{\mathrm{s}}(x, s)=\frac{C_{0}}{s+\mu}$

Substitution of Eq. (6) into Eq. (5) yields

$u \frac{\partial \bar{C}(x, s)}{\partial x}+(s+Y) \bar{C}(x, s)=\frac{E C_{0}}{s+\mu}$

The Laplace transform of Eq. (7) with respect to distance $x$ leads to

$p u \overline{\bar{C}}(p, s)+(s+Y) \overline{\bar{C}}(p, s)=\frac{E C_{0}}{s+\mu} \frac{1}{p}$

where $\overline{\bar{C}}(p, s)$ is the Laplace transform of $\bar{C}(x, s)$ with respect to the distance $x ; s$ and $p$ are known as the arguments of the Laplace transform with respect to $t$ and $x$, respectively. These arguments may be complex numbers with real parts that are sufficiently large to make the Laplace transforms converge, but in the present context these arguments are simply real nonnegative numbers. Rearranging the terms and dividing both sides by the summation of coefficients of the variable $\overline{\bar{C}}(p, s)$ gives

$\overline{\bar{C}}(p, s)=\frac{E C_{0}}{s+\mu}\left[\frac{1}{u p^{2}+(s+Y) p}\right]$ 
In order to facilitate the use of standard formulas of the inverse Laplace transform, Eq. (9a) can be rewritten as

$$
\begin{aligned}
\overline{\bar{C}}(p, s)= & \frac{E C_{0}}{u(s+\mu)} \\
& \times \frac{1}{\left[p+\frac{1}{2 u}(s+Y)\right]^{2}-\left[\frac{1}{2 u}(s+Y)\right]^{2}}
\end{aligned}
$$

The inverse Laplace transform of $\overline{\bar{C}}(p, s)$ from the $p$-domain to the $x$-domain can be obtained by comparing Eq. (9b) with the following formula of the inverse Laplace transform $\left(L^{-1}\right)$

$$
\begin{gathered}
L^{-1}\left[\frac{1}{(p+b)^{2}-a^{2}}\right]=\frac{e^{-b x} \sinh a x}{a} \\
=\frac{e^{-b x}}{a}\left(\frac{e^{a x}-e^{-a x}}{2}\right) \quad(p>b)
\end{gathered}
$$

as

$$
\begin{aligned}
\bar{C}(x, s)= & \frac{E C_{0} \exp \left[-\frac{x}{2 u}(s+Y)\right]}{(s+\mu)(s+Y)} \\
& \left\{\exp \left[\frac{x}{2 u}(s+Y)\right]-\exp \left[-\frac{x}{2 u}(s+Y)\right]\right\}
\end{aligned}
$$

This equation can be further simplified as

$$
\bar{C}(x, s)=\frac{E C_{0}}{(s+\mu)(s+Y)}\left\{1-\exp \left[-\frac{x}{u}(s+Y)\right]\right\}
$$

Eq. (11b) is the Laplace transformed solution of $C(x, t)$ and can be employed to determine the four parameters $\mu, C_{0}, E$, and $\alpha$. The characteristics of the curves of concentration distributions are generally described by statistical measures such as mean, variance, skewness, and kurtosis, which can be determined using measured data. Therefore, the idea behind the parameter estimation using the moment method is to first derive a set of moment equations for the statistical measures depending on the number of the parameters. Then, the values of the parameters are determined by equating the moment equations with the corresponding data-based statistical measures.

\subsection{Moment equations of parameter estimation}

Four equations are needed for evaluating the four parameters involved in the overland flow and solute transport equations. The four equations can be found by the first through fourth order derivatives of Eq. (11b) with respect to argument $s$

$$
\begin{aligned}
\frac{\partial \bar{C}(x, s)}{\partial s}= & -\frac{E C_{0}(2 s+Y+\mu)}{[(s+\mu)(s+Y)]^{2}}\left\{1-\exp \left[-\frac{x}{u}(s+Y)\right]\right\} \\
& +\frac{E C_{0}}{(s+\mu)(s+Y)}\left\{\frac{x}{u} \exp \left[-\frac{x}{u}(s+Y)\right]\right\}
\end{aligned}
$$

Differentiating both sides of Eq. (12) yields

$$
\begin{aligned}
\frac{\partial^{2} \bar{C}(x, s)}{\partial s^{2}}= & \left\{\frac{2 E C_{0}(2 s+Y+\mu)^{2}}{[(s+\mu)(s+Y)]^{3}}-\frac{2 E C_{0}}{[(s+\mu)(s+Y)]^{2}}\right\} \\
& \times\left\{1-\exp \left[-\frac{x}{u}(s+Y)\right]\right\} \\
& -\frac{\mathrm{E} C_{0} x}{u[(s+\mu)(s+Y)]}\left[\frac{2(2 s+Y+\mu)}{(s+\mu)(s+Y)}+\frac{x}{u}\right] \\
& \times \exp \left[-\frac{x}{u}(s+Y)\right](14)
\end{aligned}
$$

Differentiating both sides of Eq. (13) with respect to $s$ gives

$$
\begin{aligned}
& \frac{\partial^{3} \bar{C}(x, s)}{\partial s^{3}} \\
& =6 E C_{0}\left\{\frac{2(2 s+Y+\mu)}{[(s+\mu)(s+Y)]^{3}}-\frac{(2 s+Y+\mu)^{3}}{[(s+\mu)(s+Y)]^{4}}\right\} \\
& \times\left\{1-\exp \left[-\frac{x}{u}(s+Y)\right]\right\} \\
& +\frac{E C_{0} x}{u}\left\{\frac{6(2 s+Y+\mu)^{2}}{[(s+\mu)(s+Y)]^{3}}+\frac{3(2 s+Y+\mu) x}{u[(s+\mu)(s+Y)]^{2}}\right. \\
& \left.-\frac{6}{[(s+\mu)(s+Y)]^{2}}+\frac{x^{2}}{u^{2}} \frac{1}{(s+\mu)(s+Y)}\right\} \\
& \times \exp \left[-\frac{x}{u}(s+Y)\right]
\end{aligned}
$$


Differentiating both sides of Eq. (14) with respect to $s$ leads to

$$
\begin{aligned}
\frac{\partial^{4} \bar{C}(x, s)}{\partial s^{4}}= & 24 E C_{0}\left\{\frac{(2 s+Y+\mu)^{4}}{[(s+\mu)(s+Y)]^{5}}\right. \\
& \left.-\frac{3(2 s+Y+\mu)^{2}}{[(s+\mu)(s+Y)]^{4}}+\frac{1}{[(s+\mu)(s+Y)]^{3}}\right\} \bullet \\
& \times\left\{1-\exp \left[-\frac{x}{u}(s+Y)\right]\right\} \\
& +\frac{6 E C_{0} x}{u}\left\{\frac{-4(2 s+Y+\mu)^{3}}{[(s+\mu)(s+Y)]^{4}}\right. \\
& -\frac{2(2 s+Y+\mu)^{2} x}{u[(s+\mu)(s+Y)]^{3}}+\frac{8(2 s+Y+\mu)}{[(s+\mu)(s+Y)]^{3}} \\
& +\frac{2 x}{u[(s+\mu)(s+Y)]^{2}}-\frac{2(2 s+Y+\mu) x^{2}}{3 u^{2}[(s+\mu)(s+Y)]^{2}} \\
& \left.-\frac{x^{3}}{6 u^{3}} \frac{1}{(s+\mu)(s+Y)}\right\} \exp \left[-\frac{x}{u}(s+Y)\right]
\end{aligned}
$$

By the definition of the Laplace transform, which actually is the moment generating function (Deng, 2002), the $n$th derivative of $\bar{C}(x, s)$ with respect to the transform argument $s$ can be written as

$\frac{\partial^{n} \bar{C}(x, s)}{\partial s^{n}}=\int_{0}^{\infty} \frac{\partial^{n}\left(e^{-s t}\right)}{\partial s^{n}} C(x, t) \mathrm{d} t=\int_{0}^{\infty}(-t)^{n} e^{-s t} C(x, t) \mathrm{d} t$

To simplify the definite integral in Eq. (16a), $s$ is commonly set to zero. Then,

$\left.\frac{\partial^{n} \bar{C}(x, s)}{\partial s^{n}}\right|_{s=0}=(-1)^{n} \int_{0}^{\infty} t^{n} C(x, t) \mathrm{d} t$

In practice the upper limit of the integration on the right-hand-side (RHS) takes on a fixed value of time corresponding to $C(x, t)=0$. Therefore, the RHS of Eq. (16b) can be estimated using measured time versus concentration data and a numerical integration method. It is apparent that the $n$th temporal moment $M_{n}$ of the solute transport rate distribution about the origin can be found from Eq. (16b) as follows:

$M_{n}=\int_{0}^{\infty} t^{n} C(x, t) \mathrm{d} t=\left.(-1)^{n} \frac{\partial^{n} \bar{C}(x, s)}{\partial s^{n}}\right|_{s=0}$

Substituting Eqs. (12)-(15) into Eq. (17) yields the first to fourth order moment equations for the four parameters as follows:

$$
\begin{aligned}
M_{1}= & -\left.\frac{\partial \bar{C}(x, s)}{\partial s}\right|_{s=0}=\frac{E C_{0}(\mu+Y)}{(\mu Y)^{2}}\left[1-\exp \left(-\frac{x}{u} Y\right)\right] \\
& -\frac{E C_{0} x}{\mu Y u} \exp \left(-\frac{x}{u} Y\right) \\
M_{2}= & \left.\frac{\partial^{2} \bar{C}(x, s)}{\partial s^{2}}\right|_{s=0} \\
= & {\left[\frac{2 E C_{0}(\mu+Y)^{2}}{(\mu Y)^{3}}-\frac{2 E C_{0}}{(\mu Y)^{2}}\right]\left[1-\exp \left(-\frac{x}{u} Y\right)\right] } \\
& -\frac{E C_{0} x}{u(\mu Y)}\left[\frac{2(\mu+Y)}{\mu Y}+\frac{x}{u}\right] \exp \left(-\frac{x}{u} Y\right) \\
M_{3}= & -\left.\frac{\partial^{3} \bar{C}(x, s)}{\partial s^{3}}\right|_{s=0}=6 E C_{0}\left[\frac{(\mu+Y)^{3}}{(\mu Y)^{4}}-\frac{2(\mu+Y)}{(\mu Y)^{3}}\right] \\
& \times\left[1-\exp \left(-\frac{x}{u} Y\right)\right]-\frac{E C_{0} x}{u}\left[\frac{6(\mu+Y)^{2}}{(\mu Y)^{3}}\right. \\
& \left.+\frac{3(\mu+Y) x}{u(\mu Y)^{2}}-\frac{6}{(\mu Y)^{2}}+\frac{x^{2}}{u^{2}(\mu Y)}\right] \exp \left(-\frac{x}{u} Y\right) \\
& {[19) } \\
&
\end{aligned}
$$

$$
\begin{aligned}
M_{4}= & \left.\frac{\partial^{4} \bar{C}(x, s)}{\partial s^{4}}\right|_{s=0}=24 E C_{0}\left[\frac{(\mu+Y)^{4}}{(\mu Y)^{5}}-\frac{3(\mu+Y)^{2}}{(\mu Y)^{4}}\right. \\
& \left.+\frac{1}{(\mu Y)^{3}}\right]\left[1-\exp \left(-\frac{x}{u} Y\right)\right]+\frac{6 E C_{0} x}{u} \\
& \times\left[\frac{-4(\mu+Y)^{3}}{(\mu Y)^{4}}-\frac{2(\mu+Y)^{2} x}{u(\mu Y)^{3}}+\frac{8(\mu+Y)}{(\mu Y)^{3}}\right. \\
& \left.+\frac{2 x}{u(\mu Y)^{2}}-\frac{2(\mu+Y) x^{2}}{3 u^{2}(\mu Y)^{2}}-\frac{x^{3}}{6 u^{3}(\mu Y)}\right] \exp \left[-\frac{x}{u} Y\right]
\end{aligned}
$$

In practical applications the moments $M_{1}-M_{4}$ should be normalized by dividing the moments by the transported weight of the solute $M_{\mathrm{W}}=\Sigma C\left(t_{i}\right) \Delta t$, where $C\left(t_{i}\right)$ is the average measured transport rate of solute at the outlet of the watershed in the time interval $\Delta t=t_{i}-t_{i-1}$. The four normalized 
moments, $M_{\mathrm{N} 1}=M_{1} / M_{\mathrm{W}}, M_{\mathrm{N} 2}=M_{2} / M_{\mathrm{W}}, M_{\mathrm{N} 3}=M_{3} /$ $M_{\mathrm{W}}$, and $M_{\mathrm{N} 4}=M_{4} / M_{\mathrm{W}}$ represent the mean travel time of solute, variance, skewness, and kurtosis of the concentration distribution curves, respectively.

As long as all the parameters are known in Eqs. (17)-(21), each of the moments $M_{1}-M_{4}$ can be calculated using two expressions. One expression is derived by differentiating and given in the form of Eqs. (18)-(21) and the other one is based on the definition of the moments and is expressed in the integral form of Eq. (17). The integral form of moments can be solved using measured data. Equating the moment values calculated from Eq. (17) with the corresponding differential forms in Eqs. (18)-(21) leads to four equations with four unknown parameters $\mu, C_{0}, E$, and $\alpha$. The Newton-Raphson method is employed to solve the highly nonlinear Eqs. (18)-(21) and to find values of the four parameters, $\mu$, $C_{0}, E$, and $\alpha$ according to the following tolerance limit:

$\mathrm{TOLF}=\max \left|\frac{M_{\mathrm{nc}}-M_{\mathrm{no}}}{M_{\mathrm{no}}}\right|<0.10$ for $n=1,2,3$, and 4

where $M_{\mathrm{nc}}$ is the calculated normalized moment corresponding to the right hand side of Eqs. (18)-(21) and $M_{\mathrm{no}}$ is the observed moment that is computed from Eq. (17) by numerical integration using Simpson's rule and normalized by dividing $M_{\mathrm{W}}$. Then, the overland flow and solute transport equations can be solved numerically. The results of parameter estimation are given in Table 1 . It should be noted that the value of $1.667(5 / 3)$ is directly assigned to the parameter $m$ based on the turbulent flow assumption made in the Section 2.1. The values of other four parameters in Table 1 are calculated using the four moment Eqs. (18)-(21).

Table 1

Parameters used in the overland flow and solute transport model

\begin{tabular}{llllll}
\hline Parameter & $\alpha\left(\mathrm{m}^{1 / 3} / \mathrm{s}\right)$ & $m$ & $E(1 / \mathrm{s})$ & $\mu(1 / \mathrm{s})$ & $C_{0}(\mathrm{~g} / \mathrm{s})$ \\
\hline Soil & 12.65 & 1.67 & 0.040 & 0.022 & 3.68 \\
Pavement & 16.41 & 1.67 & 0.041 & 0.022 & 3.71 \\
\hline
\end{tabular}

\subsection{Sensitivity analysis of parameters}

Sensitivity analysis estimates the rate of change in the output of a model with respect to changes in model inputs. To determine relative importance of the parameters for the new model and to test some assumptions made in this paper, sensitivity analysis of the parameters was conducted for the moments $M_{1}-M_{4}$ using the values of the parameters listed in Table 1. If the error $\Delta M_{i}(i=1,2,3$, and 4$)$ in the moments $M_{1}-M_{4}$ is defined as the difference between the values of $M_{i}$ calculated for inputs $X+\Delta X$ and $X$, then the error can be estimated using a truncated Taylor series or the absolute sensitivity $A_{\mathrm{S}}=\partial M / \partial X$ (ASCE Task Committee on Hydrology Handbook of Management Group D, 1996), i.e. $\Delta M_{i}=M_{i}(X+$ $\Delta X)-M_{i}(X) \approx(\partial M / \partial X) \Delta X$; here $\Delta X$ is the error in the model input $X$ representing the variables or parameters $\mu, C_{0}, E, \alpha$, and $f$. The error can also be expressed in relative form, $\Delta M / M$. The error $\Delta M$ is essentially the deviation sensitivity to the error $\Delta X$. The relative sensitivity $R_{\mathrm{S}}$ can be expressed as $R_{\mathrm{S}}=$ $(\partial M / \partial X)(X / M) \approx(\Delta M / M)(X / \Delta X)$ (ASCE Task Committee on Hydrology Handbook of Management Group D, 1996) for the moments $M_{1}$ through $M_{4}$ using an arbitrary but uniform constant percentage of variation in the selected parameters. Typically, $10 \%=\Delta X / X$ is used for the initial screening of sensitivity. Table 2 presents the screening of relative sensitivity $R_{\mathrm{S}}$ based on a $10 \%$ variation of the four parameters that must be determined by calibration, and a physical value, the infiltration rate $f$. The calculations of the moments $M_{1}$ through $M_{4}$ derived from Eqs. (1) and (4) are most sensitive to $\mu$, the empirical time constant that describes the exponential decline of the solute transfer rate from the soil to the overland flow during rainfall. Only the sensitivity to

Table 2

Relative sensitivity $R_{\mathrm{S}}$ of the moment equations $M_{1}$ through $M_{4}$ to parameters in the overland (soil surface) flow and solute transport

\begin{tabular}{lrrrr}
\hline Parameter & \multicolumn{1}{l}{$M_{1}$} & \multicolumn{1}{c}{$M_{2}$} & \multicolumn{1}{l}{$M_{3}$} & \multicolumn{1}{l}{$M_{4}$} \\
$X$ & & & & \\
\hline$\mu$ & -1.62 & -2.35 & -3.04 & -3.67 \\
$C_{0}$ & 1.00 & 1.00 & 1.00 & 1.00 \\
$E$ & 0.64 & 0.62 & 0.62 & 0.62 \\
$\alpha$ & 0.35 & 0.37 & 0.37 & 0.37 \\
$f$ & 0.09 & 0.09 & 0.09 & 0.09 \\
\hline
\end{tabular}


Table 3

Experimental conditions

\begin{tabular}{|c|c|c|c|c|c|c|c|}
\hline \multirow[t]{2}{*}{ Experiment } & \multicolumn{3}{|l|}{ Flume } & \multicolumn{3}{|c|}{ Rainfall Excess } & \multirow{2}{*}{$\begin{array}{l}\text { Amount of } \\
\text { Pollutant }\left(\mathrm{g} / \mathrm{m}^{2}\right)\end{array}$} \\
\hline & Length (m) & Width (m) & Depth (m) & $\begin{array}{l}\text { Intensity } \\
(\mathrm{mm} / \mathrm{h})\end{array}$ & $\begin{array}{l}\text { Duration } \\
\text { (min) }\end{array}$ & $\begin{array}{l}\text { Discharge } \\
(\mathrm{L} / \mathrm{s})\end{array}$ & \\
\hline Soil layer & 3.000 & 0.300 & 0.100 & 216 & 17.3 & 0.055 & 222.2 \\
\hline Pavement & 3.000 & 0.300 & 0.100 & 283 & 19.5 & 0.071 & 222.2 \\
\hline
\end{tabular}

$10 \%$ variation in $\mu$ increases with the order of the moment. Screened as causing the next most sensitivity is the other empirical parameter necessary to calculate the solute transport rate, $C_{0}$, the initial rate at the beginning of runoff. The calculations are less sensitive to the parameters $E$ and $\alpha$, where $E$ is the exchange rate $R$ multiplied by the empirical proportionality between the depth of overland flow and depth of the active layer $\varepsilon$. The semi-empirical parameter $\alpha$ from the kinematic wave approximation can also be derived from the roughness coefficient for the soil surface and the slope. The moments were least sensitive to the infiltration rate $f$ because the soil was wetted to field capacity before the rainfall was begun. Thus the presumption that $f$ can be treated as a constant in this initial derivation of Eqs. (1) and (4) is reasonable, but may not be so for many field conditions. Therefore, the relative screening in Table 2 implies that the two empirical parameters used to describe the solute transfer from soil to overland flow must be accurately determined by calibration to ensure accurate simulations of solute transport in runoff (Table 3).

\section{Experimental description: materials and methods}

To test the efficacy of the transport rate-based model, a laboratory test was conducted. The test involved a soil flume and a rainfall simulator.

\subsection{Characteristics of flume and soil}

A soil flume had the following dimensions: $3.000 \mathrm{~m}$ length $\times 0.300 \mathrm{~m}$ width $\times 0.120 \mathrm{~m}$ height (uncertainty of $\pm 0.0005 \mathrm{~m}$ ). Surface runoff and free percolation water were collected at the end of the flume. The soil used in the laboratory experiment was mainly composed of quartz, feldspars, quartzite, muscovite, and clay minerals. The soil was $11 \%$ clay, $10 \%$ silt and $79 \%$ sand with an uncertainty of \pm $0.5 \%$. This soil was collect from fluvial deposits on the right bank (looking downstream) of the Mondego River in the city of Coimbra, Portugal.

The original soil was sieved to remove coarse rock and organic debris, prior to being uniformly spread in the flume. To obtain a flat surface, a sharp edged straight blade that could ride on the top edge of the sidewalls of the flume was used to remove excess soil. The blade was adjusted such that the soil level in the flume equalled the retaining plate at the downstream end of the flume. Afterwards, the soil was gently tamped with a wooden block to attain a uniform dry bulk density of approximately $1100 \mathrm{~kg} / \mathrm{m}^{3}$ with an uncertainty of $\pm 6 \mathrm{~kg} / \mathrm{m}^{3}$. The resulting soil surface was smooth, lacking any significant roughness elements such as stones or plant stems. The soil had a uniform thickness of $0.10 \mathrm{~m}$ with an uncertainty of $\pm 0.005 \mathrm{~m}$. The soil surface had a slope of $10 \%$ with an uncertainty of $\pm 0.5 \%$. Standard laboratory permeability tests yielded a saturated hydraulic conductivity of $K_{\mathrm{s}}=5.7 \times 10^{-5} \mathrm{~m} / \mathrm{s}$ with a standard deviation of $1.8 \times 10^{-5} \mathrm{~m} / \mathrm{s}$, for 10 replicates. The saturated soil water content was $39 \%$ with an uncertainty of $\pm 0.5 \%$. The samples tested were obtained following exactly the same procedure as used to fill the flume and had the same bulk density.

\subsection{Characteristics of the rainfall simulator}

The basic components of the rainfall simulator were three equally spaced downward-oriented fullcone nozzles, a support structure in which the nozzles were installed, and the connections with the water supply and the pump. The spacing between the nozzles was $0.95 \mathrm{~m}$ with an uncertainty 
of $\pm 0.005 \mathrm{~m}$. The nozzles had a height of $2.20 \pm$ $0.005 \mathrm{~m}$, measured above the geometric centre of the soil surface. The working pressure on the nozzles was kept constant at $50 \mathrm{kPa}$ but the variability was not recorded. The water used in the rainfall simulation was tap water, the characteristics of which are described by de Lima et al. (2002).

\subsection{Experimental procedure}

To investigate overland flow and associated pollutant transport over the soil surface, rainstorms were simulated in the laboratory. The rainfall intensity, supplied by the rainfall simulator consisting of three static nozzles, was roughly uniformly distributed on the horizontal surface. The average rainfall intensity was $216 \mathrm{~mm} / \mathrm{h}$ (over a flume length of $3.000 \mathrm{~m}$ ). To simulate the presence of surface pollutants, $0.200 \mathrm{~kg}$ of granular salt (sodium chloride) with a mean diameter of $0.4 \mathrm{~mm}$ was applied uniformly onto the soil surface after the soil was wet to field capacity. After the salt application, the soil was subjected to a simulated rainfall event, which induced overland flow and solute transport.

Before starting the experimental runs, the soil moisture was increased to field capacity by wetting. The volumetric soil water content was approximately $20 \%$ (determined by time-domain-reflectometer measurements) just before the start of the storm events. The overland flow and pollutant transport caused by each rainfall event were measured by collecting samples, during every $10 \mathrm{~s}$ in the first the initiation of overland flow at the outlet of the flume. The concentration of the salt transported by overland flow was measured using a calibrated portable conductivity-meter. The uncertainty in the measurements was $0.0005 \mathrm{~g} / \mathrm{L}$.

\subsection{Uncertainty of the measurements}

Unavoidable errors in the measurements of salt concentrations and flow are due to several causes. Measurement errors in concentrations of salt result from the instrument errors that are inclusive of slightly nonlinear calibration data relating conductivity to salt concentrations, inexactness in temperature corrections, and occasional lags in achieving stable readings. For a constant measurement error, uncertainties in measurements of salt concentration vary from insignificant coefficients of variation of $0.0011 \%$ at the highest measurements of concentration to $50 \%$ at the lowest detection limit.

In calculating the loads of salt transported in overland flow $Q_{\mathrm{f}}$, discharge measurements based on timing the capture of water volumes are typically less accurate than conductivity measurements, but none the less more accurate than other flow measurement techniques, especially secondary or indirect measurement techniques. For the metal containers and timers used for sampling, $Q_{\mathrm{f}}=W L D / T$, where the width $W$ and length $L$ are $11.00 \pm 0.05 \mathrm{~cm}$ and the depth $D$ is $4.50 \pm 0.05 \mathrm{~cm}$ when the flow reaches a constant level in the initial $2 \mathrm{~min}$ and samples are collected for a time $T$ of $10.0 \pm 0.5 \mathrm{~s}$. The calculus of variation can be used to perform a first order error analysis to determine the overall error in flow measurements as

$\Delta Q_{\mathrm{f}}=\sqrt{\left(\frac{\partial Q_{\mathrm{f}}}{\partial W} \Delta W\right)^{2}+\left(\frac{\partial Q_{\mathrm{f}}}{\partial L} \Delta L\right)^{2}+\left(\frac{\partial Q_{\mathrm{f}}}{\partial D} \Delta D\right)^{2}+\left(\frac{\partial Q_{\mathrm{f}}}{\partial T} \Delta T\right)^{2}}$

$2 \mathrm{~min}$, in metal containers placed at the downstream end of the soil flume. Then, each minute of elapsed time involved $20 \mathrm{~s}$ of sampling and $40 \mathrm{~s}$ of no sampling until the next sampling began. To maintain a constant rainfall condition for the experiments, the sampling of solute and discharge was stopped before the rainfall simulator. The starting time for measurements corresponded, in each rainstorm event, to where

$$
\begin{aligned}
\frac{\partial Q_{\mathrm{f}}}{\partial W} & =\frac{L D}{T}, \frac{\partial Q_{\mathrm{f}}}{\partial L}=\frac{W D}{T}, \frac{\partial Q_{\mathrm{f}}}{\partial D} \\
& =\frac{W L}{T}, \text { and } \frac{\partial Q_{\mathrm{f}}}{\partial T}=-\frac{W L D}{T^{2}}=-\frac{Q_{\mathrm{f}}}{T}
\end{aligned}
$$

Substituting Eq. (24) into Eq. (23) and noting $W=$ $11.0 \mathrm{~cm}, L=11.0 \mathrm{~cm}, D=4.5 \mathrm{~cm}$, and $T=10.0 \mathrm{~s}$ and $\Delta W=0.05 \mathrm{~cm}, \Delta L=0.05 \mathrm{~cm}, \Delta D=0.05 \mathrm{~cm}$, 
and $\Delta T=0.5$ s leads to

$$
\begin{aligned}
\Delta Q_{\mathrm{f}} & =Q_{\mathrm{f}} \sqrt{\left(\frac{\Delta W}{W}\right)^{2}+\left(\frac{\Delta L}{L}\right)^{2}+\left(\frac{\Delta D}{D}\right)^{2}+\left(-\frac{\Delta T}{T}\right)^{2}} \\
& =0.052 Q_{\mathrm{f}}
\end{aligned}
$$

For flow sampling of $20.0 \pm 0.5 \mathrm{~s}$ to a depth of $9.00 \pm$ $0.05 \mathrm{~cm}$, the measurement error in the flow drops to half or $0.026 Q_{\mathrm{f}}$.

The effects of rare personal mistakes in recording conductivity, water depth, or timing in these careful laboratory investigations were not quantified by replication. Instead, the agreement between the measurements and Eqs. (1) and (4) and the consistency in the measurements were used as an indication that these data are free of any significant personal mistakes.

\subsection{Comparable uncertainty in simulations of flow and concentration}

Simulation uncertainty arises from the inherent approximations used to derive the governing equations and limits on process knowledge used to formulate the equations. These uncertainties are typically lumped together and for the limited ranges of experimental and field conditions that have been measured, estimated by the difference between the limited measurements and corresponding simulations. Alternatively, a Monte Carlo analysis or a first-order error analysis as an approximation is used to determine the overall uncertainty from all input data and coefficients. In these simulations, the specification of constant rainfall intensity seems to be the only significant source of simulation uncertainty because the laboratory flume test was otherwise well controlled. After a steady overland flow is achieved from constant intensity rainfall, then the flow is proportional to intensity and the measurement error in intensity translates directly into the simulation error of overland flow. For a $2.6 \%$ error in intensity measurements, the simulated constant flow $(0.055 \mathrm{~L} / \mathrm{s})$ has an uncertainty of $2.6 \%$ ( \pm $0.0014 \mathrm{~L} / \mathrm{s}$ ) plus what are assumed to be negligible errors from other sources.

\section{Numerical solution of the proposed model}

The objective of using a numerical method is to solve Eq. (4a) and calculate the solute transport rates, $C(x, t)$, and then the concentrations $c(x, t)$ at the outlet. To that end, the flow depth $h$ should be first calculated at each spatial grid point from the overland flow Eq. (1a).

\subsection{Numerical scheme for kinematic wave equation}

Several numerical techniques are available for the solution of the kinematic wave equation. One of the second-order finite-difference schemes is the LaxWendroff scheme (Singh, 1996). Applying the LaxWendroff scheme to Eq. (1a) yields the following finite-difference solution of the kinematic wave equation (Woolhiser, 1975; Singh, 1996):

$$
\begin{aligned}
h_{j}^{i+1} & =h_{j}^{i+1} \\
& +\Delta t\left(q_{j}^{i}-m \alpha \frac{h_{j+1}^{i^{m-1}}+h_{j-1}^{i^{m-1}}}{2} \frac{h_{j+1}^{i}+h_{j-1}^{i}}{2 \Delta x}\right) \\
& +\frac{(\Delta t)^{2}}{2} \frac{q_{j}^{i+1}-q_{j}^{i}}{\Delta t}-m \alpha \frac{(\Delta t)^{2}}{2 \Delta x}\left[\frac{h_{j+1}^{i^{m-1}}+h_{j}^{i^{m-1}}}{2}\right. \\
& \times\left(\frac{q_{j+1}^{i}+q_{j}^{i}}{2}-m \alpha \frac{h_{j+1}^{i^{m-1}}+h_{j}^{i^{m-1}}}{2} \frac{h_{j+1}^{i}-h_{j}^{i}}{\Delta x}\right) \\
& -\frac{h_{j}^{i^{m-1}}+h_{j-1}^{i^{m-1}}}{2} \\
& \left.\times\left(\frac{q_{j}^{i}+q_{j-1}^{i}}{2}-m \alpha \frac{h_{j}^{i^{m-1}}+h_{j-1}^{i^{m-1}}}{2} \frac{h_{j}^{i}-h_{j-1}^{i}}{\Delta x}\right)\right]
\end{aligned}
$$

where $q=I-f$ and superscript $i$ denotes the time step and subscript $j$ stands for the distance step; $\Delta x$ and $\Delta t$ represent the distance and time step lengths, respectively. For the downstream boundary, Eq. (26) is no longer valid and the following first-order scheme is employed:

$$
h_{j}^{i+1}=h_{j}^{i}+\Delta t\left(q_{j}^{i}-m \alpha \frac{h_{j}^{i^{m-1}}+h_{j-1}^{i^{m-1}}}{2} \frac{h_{j}^{i}-h_{j-1}^{i}}{\Delta x}\right)
$$


To ensure the numerical stability of the Lax-Wendroff scheme, the Courant condition must be satisfied, i.e.

$\frac{\Delta t}{\Delta x} \leq \frac{1}{\alpha m h^{m-1}}$

Once the flow depth is calculated, then the velocity can be determined using Eq. (1b) and the solute transport equation can be subsequently solved.

\subsection{Numerical scheme for solute transport equation}

The advection-dominated transport Eq. (4) is particularly suitable for solution using the semiLagrangian scheme, based on the method of characteristics and interpolation between grid points. The semi-Lagrangian scheme is of high accuracy and involves minimal computational effort (Holly and Preissmann, 1977; Holly and Usseglio-Polatera, 1984; Karpik and Crockett, 1997). In order to utilize the semi-Lagrangian approach, Eq. (4a) can be recast as

$\frac{\mathrm{d} C}{\mathrm{~d} t}=E C_{\mathrm{s}}-Y C$

Eq. (29) is the total derivative of the transport rate $C$ along the fluid parcel trajectory or the characteristic line defined by

$\frac{\mathrm{d} x}{\mathrm{~d} t}=u$

Integrating Eq. (29) from $\left(x_{\mathrm{d}}, t_{i}\right)$ to $\left(x_{\mathrm{a}}, t_{i+1}\right)$ along the characteristic line defined by Eq. (30) and using the explicit second-order Runge-Kutta (midpoint) method (Press et al., 1988) leads to

$$
\begin{aligned}
& C_{a}^{i+1}=C_{d}^{i}+\int_{t_{i}}^{t_{i+1}}\left[E C_{s}-Y C\right] \mathrm{d} t \\
& x_{\mathrm{d}}=x_{\mathrm{a}}+\int_{t+\Delta t}^{t} u(x, t) \mathrm{d} t=x_{\mathrm{a}}-\int_{t}^{t+\Delta t} \\
& \quad u(x, t) \mathrm{d} t=x_{\mathrm{a}}-\Delta t u\left(\frac{x_{\mathrm{d}}+x_{\mathrm{a}}}{2}, t_{n+1 / 2}\right)
\end{aligned}
$$

where subscripts $a$ and $d$ represent the arrival (at time $t+\Delta t$ ) and departure (at time $t$ ) of the parcel under consideration. Applying a trapezoidal integration rule to solve Eq. (31a) results in

$$
C_{\mathrm{a}}^{i+1}=C_{\mathrm{d}}^{i}+\frac{\Delta t}{2}\left\{\left[E C_{\mathrm{s}}-Y C\right]_{\mathrm{d}}^{i}+\left[E C_{\mathrm{s}}-Y C\right]_{\mathrm{a}}^{i+1}\right\}
$$

Eq. (4b) expressed using this scheme is

$C_{\mathrm{sd}}^{i}=C_{0} \exp (-\mu t)$

and

$C_{\mathrm{sa}}^{i+1}=C_{\mathrm{sd}}^{i} \exp (-\mu \Delta t)$

Substituting Eqs. (32) and (33) into Eq. (31c) gives

$C_{\mathrm{a}}^{i+1}=\frac{\lambda C_{\mathrm{d}}^{i}+\beta C_{\mathrm{sd}}^{i}}{1+Y}$

in which

$\lambda=1-\frac{\Delta t}{2} Y$ and $\beta=\frac{\Delta t}{2} E[1+\exp (-\mu \Delta t)]$

Eq. (34a) shows that the transport rate $C$ at each grid point $x_{\mathrm{a}}$ (the 'arrival' point) at the new time $t+\Delta t$ can be determined using the transport rate of the departure point $x_{\mathrm{d}}$ at the previous time $t$. In principle, the transport rate at all grid points at time $t$ should be known. However, the departure point $x_{\mathrm{d}}$ typically will not fall on a grid point and thus the location of the departure point $x_{\mathrm{d}}$ must be estimated first. Then, the transport rate $C$ at the departure point $x_{\mathrm{d}}$ can be interpolated using the known values of two neighbouring grid points and finally replacing that value at $x_{\mathrm{a}}$ according to Eq. (34a).

Of the multitude of interpolation methods (Press et al., 1988), cubic splines seem to be the most useful for this effort. These smooth functions do not have the significant oscillatory behavior that is characteristic of high-degree polynomial interpolators, e.g., the Lagrangian interpolator, Hermite interpolator, and similar schemes. Moreover, cubic splines have the lowest interpolation error of all fourth-order interpolating polynomials (Deng, 2002). Therefore, the commonly used natural cubic splines were adopted to perform the required interpolation. The 'natural' implies that the second derivative of the spline function is set to zero at the endpoints because this provides a boundary condition that completes the system of n-2 equations, leading to a simple tridiagonal system which can be solved easily. Once 
the solute transport rate $C$ is calculated, the solute concentration $c$ can be easily obtained from $c=C / Q_{\mathrm{f}}$.

\section{Test of proposed model and discussion of results}

To test the applicability of the kinematic-wave approximation and the transport rate-based solute transport equation, overland flow and solute transport were simulated over the infiltrating slope of the soil flume under constant rainfall for a uniform declining strength diffuse source. Parameters were estimated by using the data collected from the flume with soil and with stone pavement as summarized in Table 1. From Table 1, values of $\alpha$ can be used to determine the Manning roughness coefficient. For the $10 \%$ slope, the roughness coefficient for soil and the pavement were estimated independently as 0.025 and 0.020 from the associated $\alpha$ values of $12.65\left(\mathrm{~m}^{1 / 3} / \mathrm{s}\right)$ and $16.41\left(\mathrm{~m}^{1 / 3} / \mathrm{s}\right)$, respectively. It should be noted that under the erosion of overland flow and the impacts of rainfall soil surface becomes rough and irregular and tends to form rills although the soil surface is smooth before the start of rainfall. Therefore, the roughness coefficient of 0.025 is reasonable to the erodible soil surface due to heterogeneous soil erosion and is also consistent with the typical values recommended for turbulent overland flow on the eroded soil surfaces (Woolhiser, 1975). Therefore, there are only three parameters $\left(\mu, C_{0}\right.$, and $\left.E\right)$ to be determined in practical application of this new model. To estimate these three parameters, Eqs. (18)-(20) for the moments $M_{1}-M_{3}$ can be used.

With the three parameter values calibrated using Eqs. (18)-(20), the transport rate-based model yields hydrographs and pollutographs, as plotted in

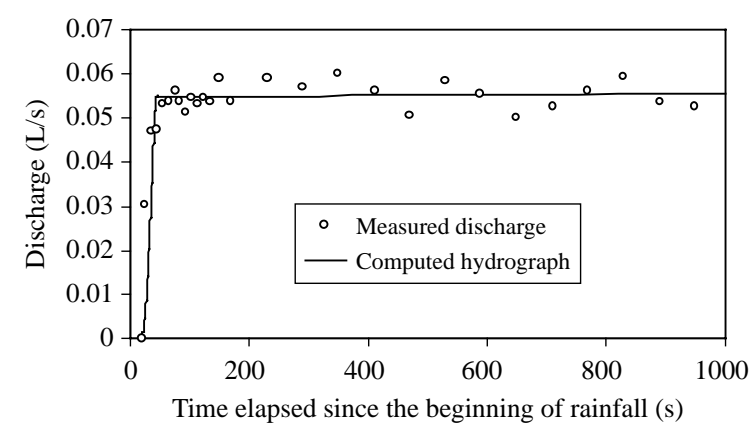

Fig. 3. Comparison between computed and measured hydrographs.

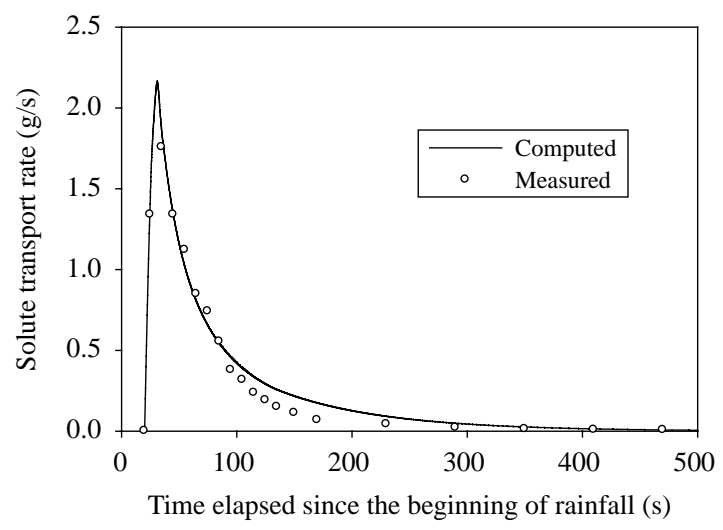

Fig. 4. Comparison between instantaneous simulated and measured (average of 10 or $20 \mathrm{~s}$ ) solute transport rates.

Figs. 3-5. Fig. 3 compares measured and computed hydrographs. Although the measured flow data vary significantly, a relative constant discharge was achieved. The measured constant flow has a mean of $0.055 \mathrm{~L} / \mathrm{s}$ and a standard deviation of $0.0028 \mathrm{~L} / \mathrm{s}$ or an uncertainty of $5.1 \%$, which is almost two times higher than that $(2.6 \%)$ of the simulated flow. The difference is most likely due to unintended variation in rainfall intensity. Fig. 4 indicates that the peak of the simulated instantaneous solute transport rate is greater than that of the measured solute transport rate. Because the measured solute transport rate at any time during the initial $2 \mathrm{~min}$ is actually the average value of the solute transport rate over the discharge collection time of $10 \mathrm{~s}$, the simulations averaged over $10 \mathrm{~s}$ are in better agreement than shown. Fig. 5 illustrates the observed and predicted pollutant

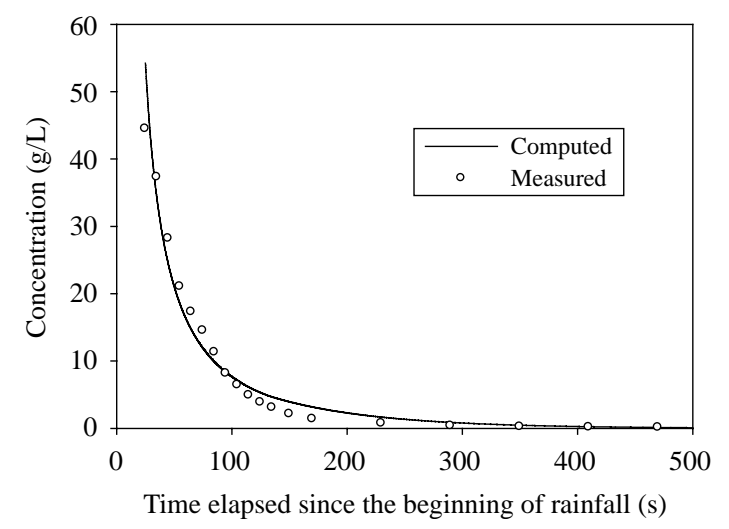

Fig. 5. Comparison between computed and measured pollutographs. 
concentration from the beginning of the rainfall. The observed concentration and the transport rate are well behaved, indicating that personal mistakes in recording the data were not obvious. The simulated instantaneous peak concentration $(54.2 \mathrm{~g} / \mathrm{L})$ is also higher than the measured average peak $(44.4 \mathrm{~g} / \mathrm{L})$ for the same reason as that of the transport rate.

\section{Conclusions}

The one-dimensional transport-rate-basedequation for overland flow over pervious, steep slopes with a declining, diffuse source of solute is not significantly different from laboratory test data. The laboratory runoff and solute transport was generated by constant rainfall intensity to achieve this proof of concept, but the measurements and thus the proof did not extend to the hydrograph recession after the rainfall ends. Furthermore, the laboratory tests minimized the effects of infiltration by wetting the soil to field capacity before the tests. This was done to initially use a constant infiltration rate in the derivation of these proposed equations.

For simulation of the increasing flow and the constant flow expected from constant rainfall (Fig. 3), the kinematic wave approximation provides outstanding simulations on the uniform $10 \%$ slope selected in the laboratory. Nevertheless, conflicts arose as to whether the flow was turbulent or laminar. The Reynolds number scaled with flow depth implies laminar flow, but it is not clear if such a shallow flow under constant impact of raindrops can really maintain a laminar regime. Calibration of the flow parameters is not precise in determining flow regime, but indicates turbulent flow that can be described by the semiempirical Manning equation. With only one test used in this initial proof of concept, the discrepancy in flow regime indications can not be resolved. However, if widespread testing does find that turbulent flow prevails, calibration of the kinematic flow approximation can be simplified with the selection of a roughness coefficient based on specific site roughness conditions. This advance will limit future model calibrations to determination of three solute transport parameters for each site, $\mu, C_{0}$, and $E$.

The calibration of an empirical description of the transfer of solute from the soil to the runoff limits this model to descriptions of known conditions and interpolation within those limits. However, for the first time it is possible to simulate realistic changes in pollutant transfer rates and concentrations. This especially includes the first flush effect that is often observed but has not been adequately simulated until now. Fig. 4 shows the effect clearly measured and simulated. Fig. 5 shows that runoff concentrations start at the highest with initiation of flow and proceed to undetectable amounts after all the salt has time to dissolve and be transferred to the runoff.

Until this new model has been tested under realistic field conditions of variable rainfall intensity and infiltration and the hydrograph recession adequately simulated, projections of the ultimate utility are difficult to make. Clearly, field predictions are not possible until better data bases are available for the selection of surface roughness coefficients and the solute transfer from the soil defined in terms of easily estimated or known physical and chemical processes that can be described mathematically. However, simulations that interpolate existing field runoff data for some simpler conditions do seem possible after calibration and careful delineation of the calibration limits by sensitivity analysis and other methods. These limited conditions include having uniform slopes steep enough for the kinematic wave approximation to be valid and a uniform declining diffuse source, both of which imply simulations of small catchments or plots of uniform slope and soils. Although the derivation does not limit the application of Eqs. (1) and (4) to uniform rainfall intensity, infiltration, and diffuse sources, the use of the moments for the necessary calibration does. While the flow parameters $m$ and $\alpha$ may be selected for some field conditions, the parameters $\mu, C_{0}$, and $E$ related to solute transfer from the soil seem for now very sitespecific and cannot be determined by extrapolation, only with calibration to measured data that we otherwise desire to predict.

\section{Acknowledgements}

The research reported in this paper was supported by the Foundation for Science and Technology of the Portuguese Ministry of Science and Technology, Lisbon, Portugal through the Post-doctoral Research 
Project SFRH/BPD/11537/2002. The host institution is the Department of Civil Engineering of the Faculty of Science and Technology at the University of Coimbra, Portugal. The laboratory experiments were also funded by the Foundation for Science and Technology (Research Projects POCTI/AGG/38957/ 2001). The writers would like to thank the two anonymous reviewers for their constructive comments and suggestions that were helpful for improving the manuscript.

\section{References}

Abbott, M.B., Refsgaard, J.C., 1996. Distributed Hydrological Modelling. Kluwer Academic Publishers, Dordrecht, The Netherlands, 121-141.

Adrian, D.D., Singh, V.P., Deng, Z.-Q., 2002. Diffusion-based semi-infinite Fourier probability distribution. J. Hydrol. Eng. 7 (2), 154-167.

Akan, A.O., 1987. Pollutant washoff by overland flow. J. Environ. Eng. 113 (4), 811-823 (See also 1993).

ASCE Task Committee on Definition of Criteria for Evaluation of Watershed Models of the Watershed Management Committee, 1993. Criteria for evaluation of watershed models. J. Irrig. Drain. Eng. 119 (3), 429-443.

ASCE Task Committee on Hydrology Handbook of Management Group D, 1996. Hydrology Handbook, second ed. ASCE.

Beven, K., 1989. Changing ideas in hydrology-the case of physically-based models. J. Hydrol. 105 (1/2), 157-172.

de Lima, J.L.M.P., 1992. Model KININF for overland flow on pervious surfaces. In: Parson, T., Abrahams, A. (Eds.), Hydraulics and Erosion Mechanics of Overland Flow. UCL Press, London, pp. 69-88.

de Lima, J.L.M.P., Singh, V.P., de Lima, M.I.P., 2002. The influence of storm movement on water erosion: storm speed and direction effects. CATENA 715, 1-18.

Deng, Z.-Q., 2002. Theoretical investigation into longitudinal dispersion in natural rivers. PhD Dissertation Lund University, Lund, Sweden.

Doherty, J., Johnston, J.M., 2003. Methodologies for calibration and predictive analysis of a watershed model. JAWRA 39 (2), 251-265.

Flanagan, D.C., Foster, G.R., 1989. Storm pattern effect on nitrogen and phosphorus losses in surface runoff. Trans ASAE 32 (2), 535-544.

Govindaraju, R.S., Erickson, L.E., 1996. Modeling of overland flow contamination due to heavy metals in shallow soil horizons. Water Resour. Res. 127, 279-305.

Holly, F.M., Preissmann, A., 1977. Accurate calculation of transport in two-dimensions. J. Hydraul. Div. 103 (11), 1259-1277.
Holly, F.M., Usseglio-Polatera, J.-D., 1984. Accurate two-dimensional simulation of advective-diffusive-reactive transport. J. Hydraul. Eng. 127 (9), 728-737.

Hubbard, R.K., Williams, R.G., Erdman, M.D., 1989a. Chemical transport from coastal plain soils under simulated rainfall: I. surface runoff, percolation, nitrate, and phosphate movement. Trans ASAE 32 (4), 1239-1249.

Hubbard, R.K., Williams, R.G., Erdman, M.D., Marti, L.R., 1989b. Chemical transport from coastal plain soils under simulated rainfall: II. movement of cyanazine, sulfometuron-methy1, and bromide. Trans ASAE 32 (4), 1250-1257.

Karpik, S.R., Crockett, S.R., 1997. Semi-Lagrangian algorithm for two-dimensional advection-diffusion equation on curvilinear coordinate meshes. J. Hydraul. Eng. 123 (5), 389-401.

Kiely, G., 1997. Environmental Engineering. McGraw-Hill International (UK) Limited, Singapore.

Martin, J.L., McCutcheon, S.C., 1999. Hydrodynamics and Transport for Water Quality Modeling. CRC Press, Inc., Boca Raton, FL pp. 7-220.

Myers, T.D., 2002. Modeling laminar sheet flow over rough surfaces. Water Resour. Res. 38 (11), 1230-1241.

Novotny, V., Olem, H., 1994. Water Quality: Prevention, Identification, and Management of Diffuse Pollution. Van Nostrand Reinhold, New York, USA.

Press, W.H., Flannery, B.P., Teukolsky, S.A., Vetterling, W.T., 1988. Numerical Recipes. Cambridge University Press, New York pp. 77-101, see also pages 615-666, 704-708.

Preti, F., 1999. Overland transport of nitrate: algorithms validation. Wat. Sci. Technol. 39 (3), 77-92.

Singh, V.P., 1996. Kinematic Wave Modeling in Water Resources: Surface-Water Hydrology. Wiley, New York pp. 897-940.

Singh, V.P., 1997. Kinematic Wave Modeling in Water Resources: Environmental Hydrology. Wiley, New York.

Singh, V.P., 1998. Entropy-based Parameter Estimation in Hydrology. Kluwer Academic, Dordrecht, the Netherlands.

Singh, V.P., 2002. Kinematic wave solution for pollutant transport over an infiltrating plane with finite-period mixing and mixing zone. Hydrol. Process. 16 (12), 2441-2477.

Singh, V.P., Deng, Z.-Q., 2003. Entropy-based parameter estimation for kappa distribution. J. Hydrol. Eng. 8 (2), 81-92.

Singh, V.P., Woolhiser, D.A., 2002. Mathematical modeling of watershed hydrology. J. Hydrol. Eng. 7 (4), 270-292.

van der Molen, W.H., Torfs, P.J.J.F., de Lima, J.L.M.P., 1995. Water depths at the upper boundary for overland flow on small gradients. J. Hydrol. 171, 93-102.

Wallach, R., Grigorin, G., Rivlin, J., 2001. A comprehensive mathematical model for transport of soil-dissolved chemicals by overland flow. J. Hydrol. 247, 85-99.

Woolhiser, D.A., 1975. Simulation of unsteady overland flow. In: Mahmood, K., Yevjevich, Y. (Eds.), Unsteady Flow in Open Channels, vol. 2. Water Resources Publications, Fort Collins, CO, pp. 485-508. 